\title{
Self-management Behavior Interventions for Type 2 Diabetes Mellitus: A Review
}

\author{
Tuan Van Nguyen ${ }^{1}$ (D), Wantonoro Wantonoro ${ }^{2 *}$ (D), Endang Koni Suryaningsih ${ }^{3}$ (D) \\ ${ }^{1}$ Department of Nursing, Faculty of Nursing and Medical Technology, Can Tho University of Medicine and Pharmacy, Can Tho \\ City, Vietnam; ${ }^{2}$ Department of Nursing, Faculty of Health Science, Universitas 'Aisyiyah, Yogyakarta, Indonesia; ${ }^{3}$ Department \\ of Midwifery, Faculty of Health Science, Universitas 'Aisyiyah, Yogyakarta, Indonesia
}

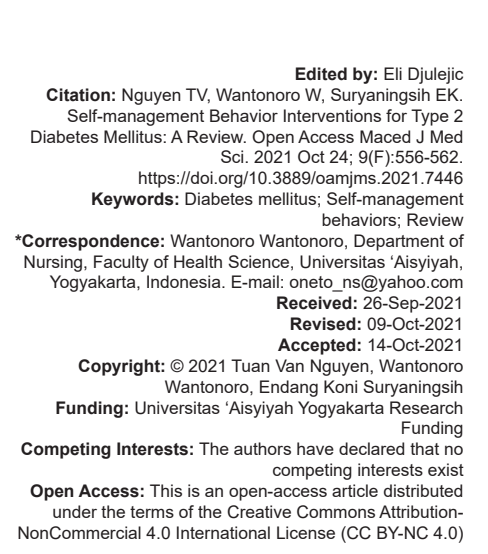

Abstract

BACKGROUND: Diabetes mellitus is chronic diseases with serious complications and reduces the quality of life of patients. Evidence based strategies to enhance diabetes self-management may prevent complications and enhance the quality of life for patients is needed.

AIM: This study to summarize the types of intervention strategies used to enhance diabetes self-management behaviors in adult individuals type 2 diabetes mellitus (T2DM)

METHOD: This study was used Literature review randomized controlled trials study. PubMed, Scopus, Web of Science, and Cochrane Library data base were used. Jadad scale checklist was used to evaluate quality appraisal included in the study.

RESULTS: Twenty-six randomized controlled trials studies were included in this review. Overall, quality of the studies was high-quality. Varied interventions included studies at different countries were found to enhance diabetes self-management behaviors in T2DM patients. In addition, almost type interventions were reported a significant enhancement diabetes self-management behaviors especially in adults with type 2 diabetes patients.

CONCLUSION: Varied self-management behavior interventions in diabetes mellitus. This review suggested for investigate best intervention to enhance diabetes self-management behaviors in different cultural for best outcomes.

\section{Introduction}

Diabetes mellitus is a dramatically increasing epidemic worldwide, with a global prevalence of approximately $8.5 \%$ of the adult population with type 2 diabetes mellitus (T2DM) [1], rate of T2DM predicted more than $60 \%$ in the Asian region by 2030 [2]. There were $85.7 \%$ fail to control the glycemic which assessment by Hemoglobin $\mathrm{HbA} 1 \mathrm{c}$ level. This serious health problem requires self-management to control the disease and associated complications. Selfmanagement includes, but is not limited to, managing a specific nutrition program, exercise program, blood glucose monitoring, and medication administration strategies [3]. The complications of DM include heart disease, stroke, kidney failure, amputation, losing vision, and neurological damage. While the major care are outpatient care, a contributing factor is self-management behaviors which are increasingly encouraged significant. The QOL of T2DM patients will enhance and reduces the risk of complications if there are strategies to enhance self-management behaviors of diabetes [4].

Diabetes self-management support decisionmaking, self-care abilities, solving problems and dynamic cooperation with the team of caring to develop patient outcomes and their QOL [5]. Diabetes self-help (DSMS) is pursuits that support diabetes patients to implement and maintain the necessary behaviors to manage their condition on a continuous basis outside of the training self-management. Despite advances in diabetes treatment, diabetes self-management still remains multiplex and hard to apply to the lifestyle in diabetes patients [6]. Furthermore, it depends to type of support provided can be behaviorsal, educational, psychosocial,or clinical strategies [5].

Based on appropriated self-care behaviors comprising medication adherence, physical activity, health nutrition, and caring feet regularly that can be manage glycemic and prevent health risks in diabetes patients. There is an evidence to suggest that social support increase diabetes self-management behaviors [7]. Self-management behaviors consist of taking medicine appropriate, testing blood glucose, foot care, do not use alcohol, and tabaco. There is an evidence that enhance diabetes-related outcomes based on good eating habits, 30 min physical exercise [8].

Many intervention studies used to enhance diabetes self-management behaviors. However, there was not review to summarize all the interventions to 
investigate the effective intervention. Therefore, this review summarized of intervention strategies used to enhance diabetes self-management behaviors in T2DM patients in randomized controlled trials (RCTs).

\section{Methods}

Literature review was conducted. Cochrane Library, PubMed Scopus, and Web of Science databases were conducted for studies published up to 2021. Search terms included those related to self-management behaviors, T2DM, and intervention studies; "self-management behaviors" OR "self-care behaviors" AND "type 2 diabetes" AND "intervention."

\section{Included and excluded studies}

Studies were included in this review following criteria; (1) randomized controlled trials (RTCs) study (2). Subjects of the study is adult patients with diagnosed of T2DM (18 years or older) (3). Included diabetes self-management behaviors or diabetes self-care behaviors in study outcome. (4) English full text. The exclusion criteria were as follows: Comments (such as blogs and electronic newspapers), reviews, letters, guidelines, and protocols.

\section{Study selection}

Two researchers screened the article titles and abstracts for eligibility. Subsequently, the full texts of the potential studies were screened to determine final eligibility for inclusion in this review. Uncertainty concerning the inclusion of the studies was checked by a third researcher.

\section{Data extraction}

The Jadad scale checklist to appraisal the methodology quality of the included studies because this is a popular scale to be used in the worldwide, higher scores presents better quality ( $\geq 4$ mean high-quality; $<3$ mean low-quality [9]. The results of this review were presented by reviewer considering: author; year of publication; country where the research was conducted; Jadad scale; setting (community, hospital); participants; interventions; follow-up; and outcomes of significant to the review question.

\section{Results}

The initial search identified 1969 articles, of which 642 were duplicates articles and removed; 1327 articles were excluded based on title and abstract, and 174 full texts were retrieved and screened. 148 articles were excluded because those were not focus to measure diabetes self-management behaviors outcomes. Finally, 26 full texts were included in this review (Figure 1).

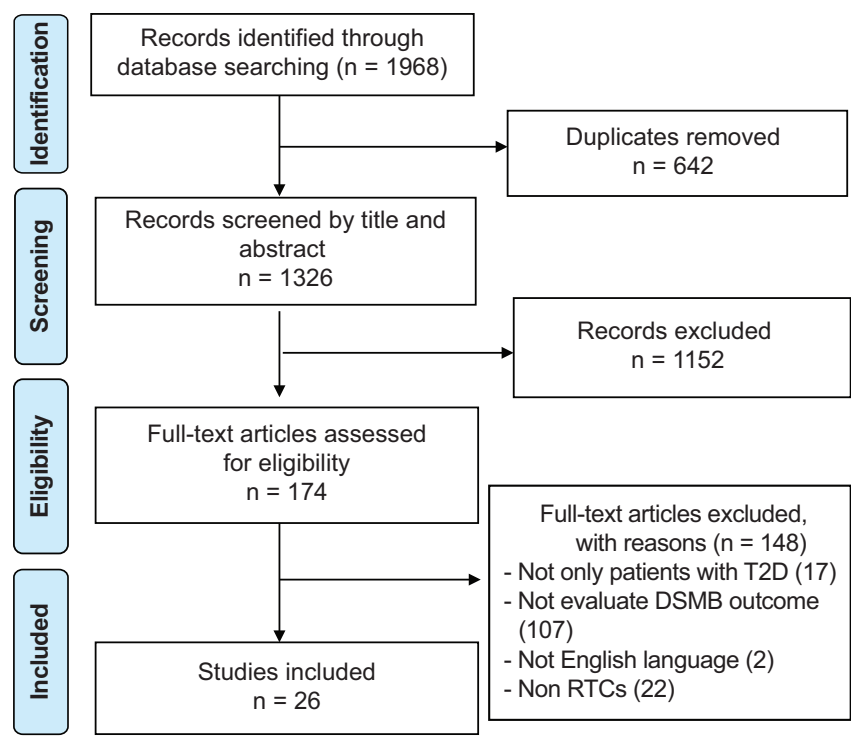

Figure 1: The process of study selection

\section{Quality of the included studies}

All of these articles showed high-quality methodology (Jadad score $>4$ ). Three among RTCs study were single-blinded [10], [11]. The quality of methodology included studies was summarized in Table 1.

\section{Characteristics of the included studies}

Of the studies in Table 2, seven were conducted in the united States of America [10], [12], [13], [14], [15], [16], [17], [18]; three were conducted in Thailand [19], [20], [21], three were conducted in Netherlands [22], [23], [24], two were conducted in China [25], [26], [27], and one study including country; Belgium [28], France [29], United Kingdom [30], German [31], Chicago [11], Cameroon [32], Iran [33], Australia [34], Africa [35], and Taiwan [36].

The sample size ranged from62 [13] to 1570 [35]. There were 16 studies to conduct in the hospital, and 10 studies were conducted in the community. The follow-up period of participant post intervention was about 3 months to 2 years. Almost studies used the 
Table 1: The level of modified Jadad quality score

\begin{tabular}{|c|c|c|c|c|c|c|c|}
\hline \multirow[t]{2}{*}{ Author (year) } & \multicolumn{7}{|l|}{ Modified Jadad scale } \\
\hline & Randomization (2) & Blinding (2) & Withdrawals and dropouts (1) & Inclusion/Exclusion criteria (1) & Adverse effects (1) & Statistical analysis (1) & Total \\
\hline Anderson (2009) & 2 & 0 & 1 & 1 & 0 & 1 & 5 \\
\hline Sacco (2009) & 2 & 0 & 1 & 1 & 0 & 1 & 5 \\
\hline Thoolen (2009) & 2 & 0 & 1 & 1 & 0 & 1 & 5 \\
\hline Mehuys (2011) & 2 & 0 & 1 & 1 & 1 & 1 & 6 \\
\hline Spencer (2011) & 2 & 2 & 1 & 1 & 1 & 1 & 8 \\
\hline Wu (2011) & 2 & 0 & 1 & 1 & 1 & 1 & 6 \\
\hline Liu (2012) & 2 & 0 & 1 & 1 & 0 & 1 & 5 \\
\hline Beverly (2013) & 2 & 0 & 1 & 1 & 0 & 1 & 5 \\
\hline Trouillound (2013) & 2 & 0 & 1 & 1 & 0 & 1 & 5 \\
\hline Van Vugt (2013) & 2 & 0 & 1 & 1 & 0 & 1 & 5 \\
\hline Forjuoh (2014) & 2 & 0 & 1 & 1 & 1 & 1 & 6 \\
\hline Mash (2014) & 2 & 0 & 1 & 1 & 0 & 1 & 5 \\
\hline Rothschild (2014) & 2 & 2 & 1 & 1 & 1 & 1 & 8 \\
\hline Steed (2014) & 2 & 0 & 1 & 1 & 1 & 1 & 6 \\
\hline Assah (2015) & 2 & 0 & 1 & 1 & 0 & 1 & 5 \\
\hline Campbell (2015) & 2 & 0 & 1 & 1 & 1 & 1 & 6 \\
\hline Sattayasomboon (2015) & 2 & 0 & 1 & 1 & 0 & 1 & 5 \\
\hline Wongrochananan (2015) & 2 & 0 & 1 & 1 & 1 & 1 & 6 \\
\hline Deng (2016) & 2 & 0 & 1 & 1 & 0 & 1 & 5 \\
\hline Shayeghian (2016) & 2 & 0 & 1 & 1 & 1 & 1 & 6 \\
\hline Hermanns (2017) & 2 & 0 & 1 & 1 & 1 & 1 & 6 \\
\hline McEwen (2017) & 2 & 0 & 1 & 1 & 0 & 1 & 5 \\
\hline Wichit (2017) & 2 & 2 & 1 & 1 & 1 & 1 & 8 \\
\hline Wroe (2017) & 2 & 0 & 1 & 1 & 1 & 1 & 6 \\
\hline Van Puffelen, et al., (2019) & 2 & 2 & 1 & 1 & 1 & 1 & 8 \\
\hline Li Cheng et al., (2021) & 2 & 2 & 1 & 1 & 1 & 1 & 8 \\
\hline
\end{tabular}

summary of diabetes self-care activities (SDSCA) to measure the outcome self-management behaviors in T2DM patients. The SDSCA Questionnaire is a popular used to assess of diabetes adherence [37].

\section{Intervention approach}

There were 22 intervention methods used for improving diabetes self-management behaviors in hospital and community setting. Firstly, nineteen interventions were applied in the hospital, included: Diabetes Self-management Consultant (CDSMC) [12]; Telephone Coaching Intervention [13]; 12-week proactive coping intervention [22]; Group Mapbased Program [14]; Therapeutic Education Program [29]; Web-based Intervention [23]; Chronic Disease Self-management Program (CDSMP) [15]; Group Diabetes Education [35]; Theoretical Based Self-management Intervention [30]; Community Based Peer Support [32]; Cognitive Behaviors Therapy (CBT) [16], [18]; Self-management Support Program (DSMP) [19]; TheAcceptance and Commitment Therapy for Diabetes Self-management [33]; Selfmanagement Oriented Education Program MEDIAS 2BSC [31]; and Family-based Diabetes Selfmanagement Intervention [17], [21]; advocating empowerment in diabetes care [27]; group-based self-management support program [24].

Secondly, seven interventions were applied in community, comprised: Community Pharmacist Intervention (28); Community Health Worker Intervention [10], [11]; Self-efficacy Enhancing Intervention Program (SEEIP) [36]; Chinese Diabetes Group Visit Model [25]; Peer Support Intervention [26]; Patient Narratives Intervention [34]; and Interactive Multi-modality Intervention (IMM) [20].

There was structural diversity of interventions, and there was considerable variation in the content of the programs. In addition, there were many different approaches to interventions to enhance self-management in T2DM patients, such as: one-to-one meeting; individual patient or group patient's education, family-oriented (patients and their family) education; peer support; telephone coaching; telephone follow-up; home visit; visual material (DVD); and web-based approach (Table 2).

\section{Outcomes}

This review studies examined a total of 22 different interventions with The Summary of Diabetes Self-Care Activities (SDSCA) to measure the outcome. There were 5 domain focuses on the outcome (diet, exercise, blood glucose testing, foot care, medication and smoking). Ten studies assessments of SDSCA were primary outcome while fifteen studies were secondary outcome.

Twenty studies [11], [13], [14], [15], [17], [18], [19], [20], [21], [22], [23], [24], [25], [26], [27], [28], [29], [30], [31], [32], [33], [34], [35], [36], [37], [38] showed enhancement in overall or some behavior of self-management in T2DM patients. However, six studies [10], [11], [12], [16], [30], [31], [35] were not statistically significant difference to compare with control group including two studies applied method used Community Health Worker Intervention [10], [11]; Diabetes self-management consultant [12], Group Diabetes Education [35], Theoretical Based Self-management [30], Self-management Oriented Education Program [31].

\section{Discussion}

The study presents the first overview of studies that summarize intervention strategies used to enhance 


\section{Table 2: Description of studies characteristics and interventions}

\begin{tabular}{|c|c|c|c|c|c|c|c|}
\hline$\overline{\text { No }}$ & Author (year), country & Setting & $\begin{array}{l}\text { Participants } \\
\text { Total (intervention/control } \\
\text { group) }\end{array}$ & Intervention & Components & Follow-up & Outcomes \\
\hline 1 & $\begin{array}{l}\text { Anderson (2009) } \\
\text { USA }\end{array}$ & Hospital & $\begin{array}{l}\text { Patients } \\
310(156 / 154)\end{array}$ & $\begin{array}{l}\text { Diabetes Self-management } \\
\text { Consultant (DSMC) }\end{array}$ & $\begin{array}{l}\text { Face-to-face consulting between patients and } \\
\text { their DSMC's }\end{array}$ & 2 years & No significant \\
\hline 2 & $\begin{array}{l}\text { Sacco (2009) } \\
\text { USA }\end{array}$ & Hospital & $\begin{array}{l}\text { Patients } \\
62(31 / 31)\end{array}$ & $\begin{array}{l}\text { Telephone Coaching } \\
\text { Intervention }\end{array}$ & $\begin{array}{l}\text { Education course } \\
1^{\text {st }} \text { three months: one phone call/week } \\
3 \text { months later: } 2 \text { weeks/one phone call }\end{array}$ & 6 months & $\begin{array}{l}\text { Significant increase } \\
\text { of diet, exercise and } \\
\text { foot care. }\end{array}$ \\
\hline 3 & $\begin{array}{l}\text { Thoolen (2009) } \\
\text { Netherlands }\end{array}$ & Hospital & $\begin{array}{l}\text { Patients } \\
197(89 / 108)\end{array}$ & $\begin{array}{l}\text { 12-week Proactive Coping } \\
\text { Intervention }\end{array}$ & $\begin{array}{l}\text { Individual meeting: patients discuss their } \\
\text { experiences } \\
\text { Group meeting: work on personally relevant } \\
\text { goals (exercise, diet, medication) }\end{array}$ & 12 months & $\begin{array}{l}\text { Significant increase } \\
\text { of diet, exercise, } \\
\text { and medication }\end{array}$ \\
\hline 4 & $\begin{array}{l}\text { Mehuys (2011) } \\
\text { Belgum }\end{array}$ & Community & $\begin{array}{l}\text { Community pharmacist } \\
66(35 / 31) \\
\text { Patients } \\
288(153 / 135)\end{array}$ & $\begin{array}{l}\text { Community Pharmacist } \\
\text { Intervention }\end{array}$ & $\begin{array}{l}\text { Education T2DM and complications } \\
\text { Correct taking and adherence of medication } \\
\text { Healthy lifestyle education } \\
\text { Reminders annual eye and foot care }\end{array}$ & 6 months & $\begin{array}{l}\text { Significant increase } \\
\text { of exercise and foot } \\
\text { care }\end{array}$ \\
\hline 5 & $\begin{array}{l}\text { Spencer (2011) } \\
\text { The USA }\end{array}$ & Community & $\begin{array}{l}\text { Patients } \\
183(84 / 99)\end{array}$ & $\begin{array}{l}\text { Community Health Worker } \\
\text { Intervention }\end{array}$ & $\begin{array}{l}\text { Education classes } 80 \text { hours } \\
2 \text { home visits of } 60 \text { minutes each } \\
\text { Patients with their health provider visit the } \\
\text { clinic }\end{array}$ & 6 months & No significant \\
\hline 6 & $\begin{array}{l}\text { Wu (2011) } \\
\text { Taiwan }\end{array}$ & Community & $\begin{array}{l}\text { Patients } \\
145(72 / 73)\end{array}$ & $\begin{array}{l}\text { Self-efficacy- Enhancing } \\
\text { Intervention Program (SEEIP) }\end{array}$ & $\begin{array}{l}\text { Joined in counseling meeting, and telephone } \\
\text { follow-up } \\
\text { Each group of } 10-15 \text { members had four } \\
\text { weekly sessions } \\
\text { Group facilitator contacted the participants } \\
\text { by telephone at } 8 \text { and } 16 \text { weeks after the } \\
\text { intervention }\end{array}$ & 6 months & $\begin{array}{l}\text { Significantly } \\
\text { increased with the } \\
\text { outcome compare } \\
\text { with control group }\end{array}$ \\
\hline 7 & $\begin{array}{l}\text { Liu }(2012) \\
\text { China }\end{array}$ & Community & $\begin{array}{l}\text { Patients } \\
208(119 / 89)\end{array}$ & $\begin{array}{l}\text { Chinese Diabetes Group Visit } \\
\text { Model }\end{array}$ & $\begin{array}{l}\text { Introduction/feedback } \\
\text { Group education } \\
\text { Questions and answers } \\
\text { Planning and closing } \\
\text { One-on-one visits with health care providers }\end{array}$ & 12 months & $\begin{array}{l}\text { Significantly } \\
\text { increased with the } \\
\text { outcome compare } \\
\text { with control group }\end{array}$ \\
\hline 8 & $\begin{array}{l}\text { Beverly (2013) } \\
\text { USA }\end{array}$ & Hospital & $\begin{array}{l}\text { Patients } \\
135(68 / 67)\end{array}$ & Group Map-based Program & $\begin{array}{l}\text { Diabetes overview } \\
\text { Diabetes and healthy nutrition } \\
\text { Monitoring blood glucose } \\
\text { Natural course of diabetes }\end{array}$ & 12 months & $\begin{array}{l}\text { Significantly } \\
\text { increased with the } \\
\text { outcome compare } \\
\text { with control group }\end{array}$ \\
\hline 9 & $\begin{array}{l}\text { Trouillound (2013) } \\
\text { France }\end{array}$ & Hospital & $\begin{array}{l}\text { Patients } \\
120(60 / 60)\end{array}$ & $\begin{array}{l}\text { Three-day Therapeutic } \\
\text { Education Program }\end{array}$ & $\begin{array}{l}\text { Eight group meeting: interactive, } \\
\text { patient-centered, educational and problem } \\
\text { solving } \\
\text { A group consisted of } 5-8 \text { participants. }\end{array}$ & 3 months & $\begin{array}{l}\text { Significant increase } \\
\text { of diet and exercise. }\end{array}$ \\
\hline 10 & $\begin{array}{l}\text { Van Vugt (2013) } \\
\text { Netherlands }\end{array}$ & Hospital & $\begin{array}{l}\text { Patients } \\
220(110 / 110)\end{array}$ & Web-based Intervention & $\begin{array}{l}\text { Online self-management program } \\
\text { Patients are provided to gain feedback on } \\
\text { behavioral goals, action plans and assessment } \\
\text { of the completed health behaviors }\end{array}$ & 12 months & $\begin{array}{l}\text { Significantly } \\
\text { increased with the } \\
\text { outcome compare } \\
\text { with control group }\end{array}$ \\
\hline 11 & $\begin{array}{l}\text { Forjuoh (2014) } \\
\text { The USA }\end{array}$ & Hospital & $\begin{array}{l}\text { Patients } \\
196(101 / 95)\end{array}$ & $\begin{array}{l}\text { Chronic Disease Program } \\
\text { (CDSMP) }\end{array}$ & $\begin{array}{l}\text { 6-week classroom-based program } \\
\text { To teach participants to improve their making } \\
\text { plans, communicate effectively. } \\
\text { Meeting in community and clinical setting }\end{array}$ & 12 months & $\begin{array}{l}\text { Significantly } \\
\text { increased with the } \\
\text { outcome compare } \\
\text { with control group }\end{array}$ \\
\hline 12 & $\begin{array}{l}\text { Mash (2014) } \\
\text { Africa }\end{array}$ & Hospital & $\begin{array}{l}\text { Health Center } \\
34(17 / 17) \\
\text { Patients } \\
1570(710 / 860)\end{array}$ & Group Diabetes Education & $\begin{array}{l}60 \text {-min group education: diabetes, living a } \\
\text { healthy lifestyle, medication and avoiding } \\
\text { complications }\end{array}$ & 12 months & No significant \\
\hline 13 & $\begin{array}{l}\text { Rothschild (2014) } \\
\text { Chicago }\end{array}$ & Community & $\begin{array}{l}\text { Patients } \\
144(73 / 71)\end{array}$ & $\begin{array}{l}\text { Community Health Worker } \\
\text { Intervention }\end{array}$ & $\begin{array}{l}\text { Training about self-management in home for } \\
\text { patients over } 2 \text { years }\end{array}$ & 2 years & No significant \\
\hline 14 & $\begin{array}{l}\text { Steed (2014) } \\
\text { The U.K }\end{array}$ & Hospital & $\begin{array}{l}\text { Patients } \\
124(65 / 59)\end{array}$ & $\begin{array}{l}\text { Theoretical Based } \\
\text { Self-management Intervention }\end{array}$ & $\begin{array}{l}\text { Five weekly: self-regulatory and social } \\
\text { cognitive theory }\end{array}$ & 9 months & No significant \\
\hline 15 & $\begin{array}{l}\text { Assah (2015) } \\
\text { Camaroon }\end{array}$ & Hospital & $\begin{array}{l}\text { Patients } \\
200(100 / 100)\end{array}$ & $\begin{array}{l}\text { Community Based Peer } \\
\text { Support }\end{array}$ & $\begin{array}{l}\text { Based on group meetings, phone calls, } \\
\text { individual confront with peer supporters. }\end{array}$ & 6 months & $\begin{array}{l}\text { Significant increase } \\
\text { of diet, exercise, } \\
\text { blood glucose } \\
\text { monitoring, foot } \\
\text { care and smoking. }\end{array}$ \\
\hline 16 & $\begin{array}{l}\text { Campbell (2015) } \\
\text { Australia }\end{array}$ & Community & $\begin{array}{l}\text { Patients } \\
630(335 / 335)\end{array}$ & Patient Narratives Intervention & $\begin{array}{l}\text { Patients gain diabetes documents and a DVD } \\
\text { content stories related to T2Dm management }\end{array}$ & 6 months & $\begin{array}{l}\text { Significant increase } \\
\text { of diet, exercise, } \\
\text { blood glucose } \\
\text { monitoring, and foot } \\
\text { care. }\end{array}$ \\
\hline 17 & $\begin{array}{l}\text { Sattayasomboon (2015) } \\
\text { Thailand }\end{array}$ & Hospital & $\begin{array}{l}\text { Patients } \\
180(90 / 90)\end{array}$ & $\begin{array}{l}\text { Self-management Support } \\
\text { Program (DSMS) }\end{array}$ & $\begin{array}{l}\text { Education about T2DM, self-management } \\
\text { medical administration, overcome with stress } \\
\text { and problems, minimizing the self-control risks } \\
\text { and barriers }\end{array}$ & 24 weeks & $\begin{array}{l}\text { Significantly } \\
\text { increased with the } \\
\text { outcome compare } \\
\text { with control group }\end{array}$ \\
\hline 18 & $\begin{array}{l}\text { Wongrochananan (2015) } \\
\text { Thailand }\end{array}$ & Community & $\begin{array}{l}\text { Patients } \\
126(78 / 48)\end{array}$ & $\begin{array}{l}\text { Interactive Multi-modality } \\
\text { Intervention (IMM) }\end{array}$ & $\begin{array}{l}\text { Consist of, SMS, email and website with four } \\
\text { main dimension: nutrition, exercise, emotion, } \\
\text { and health care }\end{array}$ & 3 months & $\begin{array}{l}\text { Significantly } \\
\text { increased with the } \\
\text { outcome compare } \\
\text { with control group }\end{array}$ \\
\hline 19 & $\begin{array}{l}\text { Deng (2016) } \\
\text { China }\end{array}$ & Community & $\begin{array}{l}\text { Patients } \\
208(97 / 111)\end{array}$ & Peer Support Intervention & $\begin{array}{l}\text { Basic diabetes-related knowledge } \\
\text { Self-management } \\
\text { Insulin-related education } \\
\text { Skills } \\
\text { Peer support training for diabetes } \\
\text { self-management }\end{array}$ & 7 months & $\begin{array}{l}\text { Significantly } \\
\text { increased with the } \\
\text { outcome compare } \\
\text { with control group }\end{array}$ \\
\hline 20 & $\begin{array}{l}\text { Shayeghian (2016) } \\
\text { Iran }\end{array}$ & Hospital & $\begin{array}{l}\text { Patients } \\
106(53 / 53)\end{array}$ & $\begin{array}{l}\text { The Acceptance and } \\
\text { Commitment Therapy for } \\
\text { Diabetes Self-Management }\end{array}$ & $\begin{array}{l}\text { One day workshop: 2-h covers information } \\
\text { regarding the diabetes disease process }\end{array}$ & 3 months & $\begin{array}{l}\text { Significantly } \\
\text { increased with the } \\
\text { outcome compare } \\
\text { with control group }\end{array}$ \\
\hline
\end{tabular}




\begin{tabular}{|c|c|c|c|c|c|c|c|}
\hline No & Author (year), country & Setting & $\begin{array}{l}\text { Participants } \\
\text { Total (intervention/control } \\
\text { group) }\end{array}$ & Intervention & Components & Follow-up & Outcomes \\
\hline 21 & $\begin{array}{l}\text { Hermanns (2017) } \\
\text { German }\end{array}$ & Hospital & $\begin{array}{l}\text { Patients } \\
182(92 / 90)\end{array}$ & $\begin{array}{l}\text { Self-management oriented } \\
\text { education program (MEDIAS } \\
2 \text { BSC) }\end{array}$ & $\begin{array}{l}\text { Six-weekly lessons: } \\
\text { MEDIAS } 2 \text { BSC helps patients administer } \\
\text { insulin injections, monitor glycemic control }\end{array}$ & 6 months & No significant \\
\hline 22 & $\begin{array}{l}\text { McEwen (2017) } \\
\text { The USA }\end{array}$ & Hospital & $\begin{array}{l}\text { Patients and their family } \\
157 \text { dyads ( } 83 \text { dyads } / 74 \\
\text { dyads) }\end{array}$ & $\begin{array}{l}\text { Family-based Diabetes } \\
\text { Intervention }\end{array}$ & $\begin{array}{l}\text { Six 2-h educational and social support group } \\
\text { Two 2-h home visits scheduled weekly } \\
\text { Three } 20 \text {-min telephone calls scheduled } \\
\text { weekly }\end{array}$ & 6 months & $\begin{array}{l}\text { Significant increase } \\
\text { of diet and exercise }\end{array}$ \\
\hline 23 & $\begin{array}{l}\text { Wichit (2017) } \\
\text { Thailand }\end{array}$ & Hospital & $\begin{array}{l}\text { Family members } \\
140(70 / 70)\end{array}$ & $\begin{array}{l}\text { Family-oriented } \\
\text { Self-management Program }\end{array}$ & $\begin{array}{l}\text { Training classes, discussions in group, } \\
\text { telephone follow-up and visit at home }\end{array}$ & 13 weeks & $\begin{array}{l}\text { Significantly } \\
\text { increased with the } \\
\text { outcome compare } \\
\text { with control group }\end{array}$ \\
\hline 24 & $\begin{array}{l}\text { Wroe } 2017 \\
\text { USA }\end{array}$ & Hospital & $\begin{array}{l}\text { Patients } \\
140(67 / 73)\end{array}$ & $\begin{array}{l}\text { Cognitive behavioral therapy } \\
\text { (CBT) }\end{array}$ & $\begin{array}{l}\text { Considering a 'Wellbeing Group' pay attention } \\
\text { on mind and worries } \\
\text { Groups work every week for } 6 \text { weeks with } \\
\text { each group from } 6 \text { to } 12 \text { individuals }\end{array}$ & 12 months & $\begin{array}{l}\text { Significant increase } \\
\text { of diet, blood sugar } \\
\text { testing and feet care }\end{array}$ \\
\hline 25 & $\begin{array}{l}\text { Van Puffelen, } \\
\text { et al., (2019) } \\
\text { Netherlands. }\end{array}$ & Hospital & $\begin{array}{l}\text { Patients } \\
168(82 / 86)\end{array}$ & $\begin{array}{l}\text { Group-based } \\
\text { self-management support } \\
\text { program }\end{array}$ & $\begin{array}{l}\text { Group-based self-management support } \\
\text { program for people diagnosed with type } \\
2 \text { diabetes ( } 1-3 \text { years post diagnosis) on } \\
\text { diabetes self-care, distress and cognitions }\end{array}$ & 6 Six month & $\begin{array}{l}\text { intervention } \\
\text { group showed a } \\
\text { significantly higher } \\
\text { increase in physical } \\
\text { activity and diit }\end{array}$ \\
\hline 26 & $\begin{array}{l}\text { Li Cheng et al., (2021) } \\
\text { China }\end{array}$ & Hospital & $\begin{array}{l}\text { Patients } \\
242(121 / 121)\end{array}$ & $\begin{array}{l}\text { Empowerment-based } \\
\text { transitional care program }\end{array}$ & Advocating empowerment in diabetes care & 6-week & $\begin{array}{l}\text { participants in the } \\
\text { intervention group } \\
\text { showed significant } \\
\text { improvements on } \\
\text { empowerment level }\end{array}$ \\
\hline
\end{tabular}

diabetes self-management behaviors in T2DM. There are 26 studies from 14 different countries summarizing 22 different intervention strategies for enhancing self-management behaviors in T2DM patients.

The result highlighted that the efficacy of the diabetes self-management behaviors of the patients with T2DM is not consistent. The results of interventions used to enhance diabetes self-management behaviors are very different although the same instrument outcome was used. There are five interventions that show an unchanged in diabetes self-management behaviors outcomes. Although there are many different approaches to enhance self-management behavior in T2DM patients, group-based, family-based and peer support interventions show more effectively [11], [13], [14], [15], [17]. [18], [19], [20], [21], [22], [23], [25], [26], [28], [29], [32], [33], [34], [36], [38]. Most studies (20 articles) were significant but did not report the detail of subscale in instrument; they displayed only the total score and its relevance between intervention and outcome. Most of the studies assessed the diabetes self-management behavior as the secondary outcome, so the subscale was not be described in detail. Overall, almost measurement outcome have been met one time; only two interventions meet two times. The findings from this review suggest many studies with different populations or time are needed to conduct to examine the effectiveness of these programs.

One of the strengths of this review, used Jadad scale, the most widely used scale in the word, to provide methodological quality of the study examined [39]. All of these articles in this review show methodology with high-quality (score $>4$ ). The use of this scale in future reviews, or in similar studies, would facilitate identification of progress in scientific evidence concerning the intervention to enhance diabetes self-management behaviors in T2DM patients.
Moreover, we employed a very thorough search strategy to identify most of the current studies of intervention on diabetes self-management behaviors in T2DM patients. However, it is nevertheless possible that some studies may only have been published in local databases and were not, therefore, included in this review.

This review has several limitations. First, this review had limitations in the synthesis of findings. This study shows only interventions that have been used to enhance diabetes self-management behavior in adults T2DM, but do not analyze and compare the effects of interventions. A meta-analysis is needed to better understand this issue.

\section{Conclusion}

This review provides a significant contribution related to intervention methods used to enhance diabetes self-management behaviors in T2DM patients. However, the effectiveness of these interventions is varied. This review suggested for investigate best intervention to enhance diabetes self-management behaviors in different cultural for best outcomes. In addition, developing hospital and community administrative services to improve diabetes self-management behaviors for this population recommended for future studies.

\section{References}

1. World Health Organization. Global Report on Diabetes. Geneva: World Health Organization; 2016. 
2. Abdullah N, Attia J, Oldmeadow C, Scott RJ, Holliday EG. The architecture of risk for Type 2 diabetes: Understanding Asia in the context of global findings. Int J Endocrinol 2014;2014:593982. https://doi.org/10.1155/2014/593982

\section{PMid:24744783}

3. American Diabetes Association. Executive summary: Standards of medical care in diabetes-2014. Diabetes Care 2014;37 Suppl 1:S5-13. https://doi.org/10.2337/dc14-S005 PMid:24357214

4. Franek J. Self-management support interventions for persons with chronic disease: An evidence-based analysis. Ont Health Technol Assess Ser. 2013;13(9):1-60.

PMid:24194800

5. Funnell MM, Brown TL, Childs BP, Haas LB, Hosey GM, Jensen B, et al. National standards for diabetes selfmanagement education. Diabetes Care. 2014;37 Suppl 1:S8794. https://doi.org/10.2337/dc08-S097

PMid:18165344

6. Odegard PS, Capoccia K. Medication taking and diabetes. Diabetes Educ. 2007;33(6):1014-29. https://doi. org/10.1177/0145721707308407

PMid: 18057270

7. Strom JL, Egede LE. The impact of social support on outcomes in adult patients with Type 2 diabetes: A systematic review. Curr Diab Rep. 2012;12(6):769-81. https://doi.org/10.1007/ s11892-012-0317-0

\section{PMid:22949135}

8. Centers for Disease Control and Prevention. National Diabetes Statistics Report; Estimates of Diabetes and Its Burden in the United States; 2020. Available form: https://www.cdc.gov/ diabetes/data/statistics-report/index.html [Last accessed on 2021 Jan 20].

9. Oremus M, Wolfson C, Perrault A, Demers L, Momoli F, Moride $Y$. Interrater reliability of the modified Jadad quality scale for systematic reviews of Alzheimer's disease drug trials. Dement Geriatr Cogn Disord. 2001;12(3):232-6. https://doi. org/10.1159/000051263

PMid:11244218

10. Spencer MS, Rosland AM, Kieffer EC, Sinco BR, Valerio M, Palmisano G, et al. Effectiveness of a community health worker intervention among African American and Latino adults with Type 2 diabetes: A randomized controlled trial. Am J Public Health. 2011;101(12):2253-60. https://doi.org/10.2105/ AJPH.2010.300106

PMid:21680932

11. Rothschild SK, Martin MA, Swider SM, Lynas CM, Janssen I, Avery EF, et al. Mexican american trial of community health workers: A randomized controlled trial of a community health worker intervention for mexican americans with Type 2 diabetes mellitus. Am J Public Health. 2014;104(8):1540-8. https://doi. org/10.2105/AJPH.2013.301439

PMid:23947316

12. Anderson RM, Funnell MM, Aikens JE, Krein SL, Fitzgerald JT, Nwankwo R, et al. Evaluating the efficacy of an empowermentbased self-management consultant intervention: Results of a two-year randomized controlled trial. Ther Patient Educ. 2009;1(1):3-11. https://doi.org/10.1051/tpe/2009002 PMid:20076768

13. Sacco WP, Malone JI, Morrison AD, Friedman A, Wells K. Effect of a brief, regular telephone intervention by paraprofessionals for Type 2 diabetes. J Behav Med. 2009;32(4):349-59. https:// doi.org/10.1007/s10865-009-9209-4.

PMid:19365719

14. Beverly EA, Fitzgerald SM, Brooks KM, Hultgren BA, Ganda OP, Munshi $\mathrm{M}$, et al. Impact of reinforcement of diabetes self-care on poorly controlled diabetes: A randomized controlled trial. Diabetes Educ. 2013;39(4):504-14. https://doi. org/10.1177/0145721713486837

PMid:23640303

15. Forjuoh SN, Ory MG, Jiang L, Vuong AM, Bolin JN. Impact of chronic disease self-management programs on Type 2 diabetes management in primary care. World $\mathrm{J}$ Diabetes. 2014;5(3):407-14. https://doi.org/10.4239/wjd.v5.i3.407 PMid:24936263

16. Inouye J, Li D, Davis J, Arakaki R. Psychosocial and clinical outcomes of a cognitive behavioral therapy for asians and pacific islanders with Type 2 diabetes: A randomized clinical trial. Hawaii J Med Public Health. 2015;74(11):360-8.

PMid:26568899

17. McEwen MM, Pasvogel A, Murdaugh C, Hepworth J. Effects of a family-based diabetes intervention on behavioral and biological outcomes for Mexican American adults. Diabetes Educ. 2017;43(3):272-85. https://doi.org/10.1177/0145721717706031 PMid:28447545

18. Wroe AL, Rennie EW, Sollesse S, Chapman J, Hassy A. Is cognitive behavioural therapy focusing on depression and anxiety effective for people with long-term physical health conditions? A controlled trial in the context of Type 2 diabetes mellitus. Behav Cogn Psychother. 2017;46(2):129-47. https:// doi.org/10.1017/S1352465817000492

PMid:28849755

19. Sattayasomboon $\mathrm{Y}$, Jiammjarasrungsi $\mathrm{W}$, Lertmaharit $\mathrm{S}$, Lohsoonthorn V. Effectiveness of a self-management support program for Type 2 diabetes mellitus in public health centers in Bangkok, Thailand: Randomized controlled trial. Gerontologist. 2015;55:71-2.

20. Wongrochananan S, Tuicomepee A, Buranarach M, Jiamjarasrangsi $W$. The effectiveness of interactive multimodality intervention on self-management support of Type 2 diabetic patients in Thailand: A cluster-randomized controlled trial. Int J Diabetes Dev Ctries. 2015;35:230-6.

21. Wichit N, Mnatzaganian G, Courtney M, Schulz P, Johnson M. Randomized controlled trial of a family-oriented self-management program to improve self-efficacy, glycemic control and quality of life among Thai individuals with Type 2 diabetes. Diabetes Res Clin Pract. 2017;123:37-48. https://doi. org/10.1016/j.diabres.2016.11.013

\section{PMid:27918976}

22. Thoolen BJ, de Ridder D, Bensing J, Gorter K, Rutten G. Beyond good intentions: The role of proactive coping in achieving sustained behavioural change in the context of diabetes management. Psychol Health. 2009;24(3):237-54. https://doi. org/10.1080/0887044070186450

PMid:20204991

23. van Vugt $M$, de Wit $M$, Hendriks $S H$, Roelofsen $Y$, Bilo $H J$, Snoek FJ. Web-based self-management with and without coaching for Type 2 diabetes patients in primary care: Design of a randomized controlled trial. BMC Endocr Disord. 2013;13:53. https://doi.org/10.1186/1472-6823-13-53

PMid:24238104

24. van Puffelen AL, Rijken M, Heijmans MJ, Nijpels G, Schellevis FG, Diacourse Study Group. Effectiveness of a self-management support program for Type 2 diabetes patients in the first years of illness: Results from a randomized controlled trial. PLoS One. 2019;14(6):e0218242. https://doi.org/10.1371/ journal.pone.0218242 PMid:31247039

25. Liu S, Bi A, Fu D, Fu H, Luo W, Ma X, et al. Effectiveness of using group visit model to support diabetes patient self-management in rural communities of Shanghai: A randomized controlled trial. BMC Public Health. 2012;12:1043. https://doi. 
org/10.1186/1471-2458-12-1043

\section{PMid:23198694}

26. Deng K, Ren Y, Luo Z, Du K, Zhang X, Zhang Q. Peer support training improved the glycemic control, insulin management, and diabetic behaviors of patients with Type 2 diabetes in rural communities of Central China: A randomized controlled trial. Med Sci Monit. 2016;22:267-75. https://doi.org/10.12659/ msm.895593

PMid:26808489

27. Cheng L, Sit JW, Choi KC, Chair SY, Li X, Wu Y, et al. The effects of an empowerment-based self-management intervention on empowerment level, psychological distress, and quality of life in patients with poorly controlled Type 2 diabetes: A randomized controlled trial. Int J Nurs Stud. 2021;116:103407. https://doi. org/10.1016/j.ijnurstu.2019.103407

PMid:31679744

28. Mehuys E, van Bortel $L$, de Bolle $L$, van Tongelen I, Annemans L, Remon JP, et al. Effectiveness of a community pharmacist intervention in diabetes care: $A$ randomized controlled trial. J clin Pharm Ther. 2011;36(5):602-13. https:// doi.org/10.1111/j.1365-2710.2010.01218.x PMid:21143256

29. Trouilloud D, Regnier J. Therapeutic education among adults with Type 2 diabetes: Effects of a three-day intervention on perceived competence, self-management behaviours and glycaemic control. Glob Health Promot. 2013;20 Suppl 2:94-8. https://doi.org/10.1177/1757975913483331 PMid:23678503

30. Steed L, Barnard M, Hurel S, Jenkins C, Newman S. How does change occur following a theoretically based self-management intervention for Type 2 diabetes. Psychol Health Med. 2014;19(5):536-46. https://doi.org/10.1080/13548506.2013.845 301

\section{PMid:24111492}

31. Hermanns N, Ehrmann D, Schall S, Maier B, Haak T, Kulzer $B$. The effect of an education programme (MEDIAS 2 BSC) of non-intensive insulin treatment regimens for people with Type 2 diabetes: A randomized, multi-centre trial. Diabetic Med. 2017;34(8):1084-91. https://doi.org/10.1111/dme.13346 PMid:28257159

32. Assah F, Atanga E, Enoru S, Sobngwi E, Mbanya J. Community-based peer support significantly improves metabolic control in people with Type 2 diabetes in Yaoundé, Cameroon. Diabetic Med. 2015;32(7):886-9. https://doi.org/10.1111/ dme.12720

PMid:25659589

33. Shayeghian $Z$, Hassanabadi $H$, Aguilar-Vafaie ME, Amiri P, Besharat MA. A randomized controlled trial of acceptance and commitment therapy for Type 2 diabetes management: The moderating role of coping styles. PLoS One. 2016;11(12):e0166599. https://doi.org/10.1371/journal. pone.0166599 PMid:27907074

34. Campbell T, Dunt D, Fitzgerald JL, Gordon I. The impact of patient narratives on self-efficacy and self-care in Australians with Type 2 diabetes: Stage 1 results of a randomized trial. Health Promot Int. 2015;30(3):438-48. https://doi.org/10.1093/ heapro/dat058

PMid:23985247

35. Mash R, Rhode H, Zwarenstein M, Rollnick S, Lombard C, Steyn $\mathrm{K}$, et al. Effectiveness of a group diabetes education programme in under-served communities in South Africa: A pragmatic cluster randomized controlled trial. Diabet Med. 2014;31(8):987-93. https://doi.org/10.1111/dme.12475 PMid:24766179

36. Wu SF, Lee MC, Liang SY, Lu YY, Wang TJ, Tung HH. Effectiveness of a self-efficacy program for persons with diabetes: Arandomized controlled trial. Nurs Health Sci. 2011;13(3):335-43. https://doi.org/10.1111/j.1442-2018.2011.00625.x PMid:21812879

37. Toobert DJ, Hampson SE, Glasgow RE. The summary of diabetes self-care activities measure: Results from 7 studies and a revised scale. Diabetes Care. 2000;23(7):943-50. https:// doi.org/10.2337/diacare.23.7.943

PMid:10895844

38. Nishimura A, Harashima SI, Fujita Y, Tanaka D, Wang Y, Liu Y, et al. Effects of structured testing versus routine testing of blood glucose in diabetes self-management: A randomized controlled trial. J Diabetes Complications. 2017;31(1):228-33. https://doi. org/10.1016/j.jdiacomp.2016.08.019 PMid:27653670

39. Liberati A, Altman DG, Tetzlaff J, Mulrow C, Gøtzsche PC loannidis JP, et al. The PRISMA statement for reporting systematic reviews and meta-analyses of studies that evaluate health care interventions: Explanation and elaboration. PLoS Med. 2009;6(7):e1000100. https://doi.org/10.1371/journal. pmed.1000100

PMid:19621070 